\title{
Efeito dos probióticos na erradicação do Helicobacter pylori: uma revisão baseada na evidência
}

Cristiana Sousa Pinto, ${ }^{1}$ Patrícia Alves, ${ }^{2}$ Joana Frasco ${ }^{3}$

\section{RESUMO}

Introdução: A infeção por Helicobacter pylori (H. pylori) constitui um importante fator de risco para inúmeras patologias gástricas, nomeadamente úlcera péptica, gastrite atrófica crónica e cancro gástrico, sendo a sua erradicação o principal objetivo terapêutico. Os probióticos podem ajudar a alcançar este objetivo, bem como desempenhar um papel positivo na redução dos efeitos laterais do tratamento.

Objetivo: Determinar o efeito dos probióticos como terapêutica adjuvante na erradicação do $H$. pylori em adultos, à luz da melhor evidência disponível.

Fontes de dados: MEDLINE e em sítios de medicina baseada na evidência.

Métodos de revisão: Pesquisa de meta-análises (MA), revisões sistemáticas (RS), ensaios clínicos aleatorizados (ECA) e normas de orientação clínica (NOC), recorrendo aos termos MeSH Helicobacter pylori e Probiotics. A pesquisa foi limitada aos artigos publicados entre janeiro de 2006 e julho de 2016, em inglês e português. Foi utilizada a Strength of Recomendation Taxonomy (SORT) para atribuição do nível de evidência (NE) e da força de recomendação (FR).

Resultados: Foram encontrados 61 artigos e selecionados, por cumprirem os critérios de inclusão, duas MA, duas RS, cinco ECA e três NOC. As MA apresentam resultados discordantes: uma evidenciou taxas de erradicação superiores com o esquema terapêutico com probióticos; a outra não demonstrou a superioridade dos probióticos quando comparados com o placebo. Ambas mostraram o seu efeito benéfico na diminuição dos efeitos laterais. As duas RS, apesar de mostrarem um efeito positivo dos probióticos nos efeitos adversos, não mostraram benefício da sua utilização para a erradicação do $H$. pylori. Todos os ECA evidenciaram taxas de erradicação da bactéria superiores no grupo medicado com probióticos. As NOC sugerem o uso de probióticos no tratamento da erradicação do $H$. pylori.

Conclusões: A adição de probióticos ao esquema de tratamento utilizado para a erradicação do $H$. pylori pode ser benéfica, uma vez que parece associar-se a taxas de erradicação superiores (FR B) com menores efeitos adversos associados (FR A).

Palavras-chave: Helicobacter pylori; Probióticos.

\section{INTRODUÇÃO}

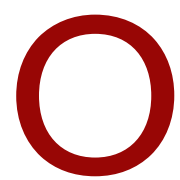

Helicobacter pylori (H. pylori) é uma bactéria espiralada gram-negativa frequentemente encontrada a nível da mucosa gástrica, estimando-se que esteja presente em pelo menos 50\% da população mundial. ${ }^{1}$ Apesar de a maioria dos indivíduos com infeção por $H$. pylori ser assintomática e nunca desenvolver qualquer processo patológico, esta infeção representa um fator de risco bem definido para inúmeras patologias gástricas, no- meadamente úlcera péptica, gastrite atrófica crónica e, menos comumente, adenocarcinoma gástrico e linfoma MALT gástrico. A sua erradicação é, portanto, fundamental para a prevenção destas complicações e constitui o principal objetivo do tratamento. ${ }^{2-3}$

Atualmente a terapêutica de primeira linha recomendada para a erradicação do H. pylorié a terapêutica

1. USF São Martinho, ACeS Tâmega II - Vale do Sousa Sul

2. UCSP Moimenta da Beira, ACeS Douro Sul

3. USF São Martinho, ACeS Tâmega II -Vale do Sousa Sul 
tripla [inibidor da bomba de protões (IBP) + claritromicina + amoxicilina ou metronidazol, durante 7-14 dias]; outras opções incluem a terapêutica sequencial e a terapêutica quadrupla. ${ }^{4}$ No entanto, devido ao aumento substancial das resistências do H. pylori aos antimicrobianos, sobretudo à claritromicina e ao metronidazol, e também devido aos efeitos adversos associados à antibioterapia (vómitos, diarreia, dor e distensão abdominal) que muitas vezes condicionam uma má adesão ao tratamento, as taxas de erradicação do H. pylori têm vindo a diminuir para valores considerados insatisfatórios. ${ }^{3,5}$ Este facto traduz a necessidade do desenvolvimento de novas estratégias para o tratamento desta infeção que não só melhorem as taxas de erradicação, mas que também reduzam a frequência de efeitos adversos. ${ }^{6}$

Recentemente a utilização de probióticos tem sido apontada como uma das possíveis soluções alternativas para esta problemática ${ }^{6}{ }^{6}$ Os probióticos são microorganismos vivos não patológicos, que quando administrados em doses adequadas beneficiam o hospedeiro através da alteração e regulação da microflora intestinal. ${ }^{7}$ Os microrganismos mais frequentemente usados nas preparações probióticas incluem o Lactobacillus spp. e o Bifidobacterium spp., o Enterococcus spp., o Streptococcus spp. e a levedura Saccharomyces boulardii (S. boulardii). ${ }^{3}$ Vários mecanismos patofisiológicos poderão estar subjacentes ao possível papel positivo dos probióticos na erradicação do H. pylori: por um lado, os probióticos podem competir diretamente com o H. pylori, produzir substâncias antimicrobianas e modular a resposta imune; por outro, podem levar a uma menor incidência de efeitos laterais e, consequentemente, a maior adesão terapêutica. ${ }^{3}$

Vários estudos se têm debruçado sobre esta temática no sentido de perceber se a utilização de probióticos como adjuvantes à terapêutica standard tem de facto um lugar na erradicação do H. pylori. No entanto, enquanto alguns estudos mostram uma melhoria significativa das taxas de erradicação e na redução dos efeitos adversos do tratamento com a associação de probióticos, outros estudos não encontraram estas associações, pelo que a utilização destas preparações na prática clínica permanece controversa. ${ }^{6}$

É, por isso, fundamental rever a evidência existente e definir em que medida a utilização de probióticos neste contexto pode ser recomendada. Esta revisão tem, assim, como objetivo determinar o efeito dos probióti- cos como terapêutica adjuvante na erradicação do $H$. pylori, bem como na redução da incidência de efeitos adversos, à luz da melhor evidência disponível.

\section{MÉTODOS}

Foi realizada uma pesquisa de meta-análises (MA), revisões sistemáticas (RS), ensaios clínicos aleatorizados e controlados (ECA) e normas de orientação clínica (NOC) publicadas entre julho de 2006 e julho de 2016, em português e inglês, nas bases de dados da National Guideline Clearinghouse, National Institute for Health and Care Excellence (NICE) Guidelines Finder, Canadian Medical Association (CMA) Practice Guidelines Infobase, The Cochrane Library, Database of Abstracts of Reviews of Effectiveness (DARE), Bandolier, EvidenceBased Medicine Online e MEDLINE, utilizando os termos MeSH Helicobacter pylori e Probiotics.

Foram selecionados artigos que incluíam indivíduos adultos (> 18 anos), não-grávidas, com diagnóstico de infeção por H. pylori, submetidos ao tratamento standard de erradicação do H. pylori, com probióticos como terapêutica adjuvante em comparação com o tratamento standard isolado ou com placebo. Os outcomes avaliados foram a taxa de erradicação da bactéria e a redução dos efeitos laterais do tratamento. Foram utilizados como critérios de exclusão: artigos duplicados, artigos de opinião, artigos de revisão clássica de tema, ensaios clínicos incluídos nas RS ou MA selecionadas e artigos que não cumpriam os critérios de inclusão no que se refere à população, intervenção, comparação ou outcomes.

Para estratificar o nível de evidência (NE) dos estudos e a força de recomendação (FR) foi utilizada a Strenght of Recomendation Taxonomy (SORT), da American Academy of Family Physicians. ${ }^{8}$

\section{RESULTADOS}

Da pesquisa efetuada foram identificados 61 artigos, dos quais foram selecionados, por cumprirem os critérios de inclusão, duas MA, duas RS, cinco ECA e três NOC. A Figura 1 representa o fluxograma da seleção dos estudos. A descrição dos artigos selecionados para revisão encontra-se resumida por categorias nos Quadros I, II, III e IV.

\section{Meta-análises}

As duas MA incluídas nesta revisão apresentam resultados discordantes entre si no que diz respeito ao papel 


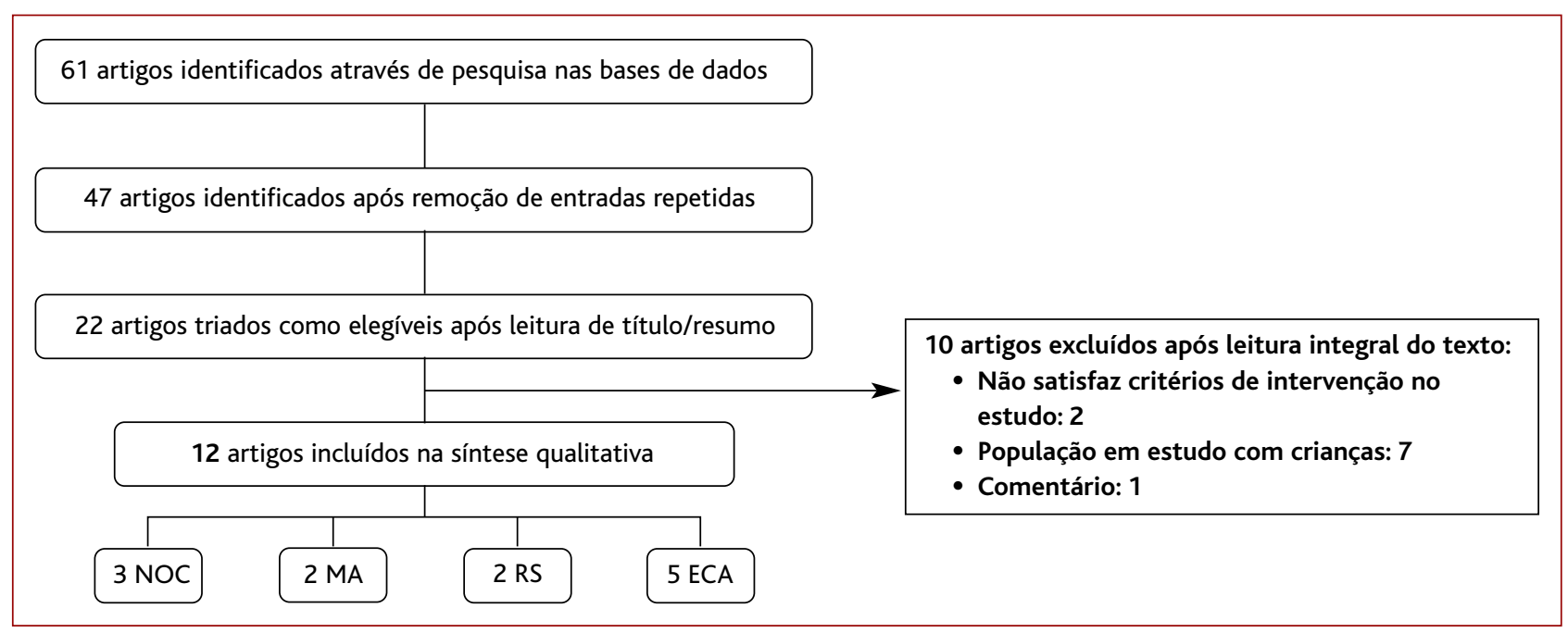

Figura 1. Fluxograma de seleção dos artigos.

Legenda: $\mathrm{NOC}=$ norma de orientação clínica; $\mathrm{MA}$ = meta-análise; $\mathrm{RS}$ = revisão sistemática; $\mathrm{ECA}$ = ensaio clínico aleatorizado e controlado.

\begin{tabular}{|c|c|c|c|}
\hline Referência & Metodologia & Resultados & NE \\
\hline $\begin{array}{l}\text { Lu, et al. } \\
(2016)^{9}\end{array}$ & $\begin{array}{l}\text { Intervenção: TS + probiótico vs TS + placebo/isolada } \\
\text { Outcome primário: Taxa de erradicação do H. pylori } \\
\text { Outcome secundário: Tolerabilidade do tratamento } \\
\text { Estudos incluídos: } 21 \mathrm{ECA}-8 \text { com grupos placebo; } \\
13 \text { sem grupos placebo }(n=3,452)\end{array}$ & $\begin{array}{l}\text { Sem diferença estatisticamente significativa na taxa } \\
\text { de erradicação do } H \text {. pylori entre os doentes com } \\
\text { suplementação com probióticos e o grupo placebo } \\
\text { (contudo, a taxa de erradicação foi superior nos } \\
\text { primeiros). } \\
\text { Os probióticos são eficazes na redução dos efeitos } \\
\text { laterais da TS. }\end{array}$ & 2 \\
\hline $\begin{array}{l}\text { Zhu, et al. } \\
(2014)^{3}\end{array}$ & $\begin{array}{l}\text { Intervenção: TTS + probiótico vs TTS + placebo/ } \\
\text { /isolada } \\
\text { Outcome primário: Taxa de erradicação do H. pylori } \\
\text { Outcome secundário: Incidência global e específica } \\
\text { de efeitos laterais } \\
\text { Estudos incluídos: } 14 \text { ECA }(n=2,259)\end{array}$ & $\begin{array}{l}\text { A suplementação com probióticos aumenta a taxa } \\
\text { de erradicação do } H \text {. pylori e diminui os efeitos } \\
\text { laterais do tratamento. }\end{array}$ & 1 \\
\hline
\end{tabular}

Legenda: $\mathrm{ECA}=$ ensaio clínico aleatorizado e controlado; $\mathrm{H}$. pylori = Helicobacter pylori; $\mathrm{NE}=$ nível de evidência; $\mathrm{TS}=$ terapêutica standard; $\mathrm{TTS}=$ terapêutica tripla standard.

dos probióticos na taxa de erradicação do H. pylori, embora ambas tenham mostrado o seu efeito benéfico na diminuição dos efeitos adversos do tratamento (Quadro I).

A MA de Lu e colaboradores, ${ }^{9}$ publicada em 2016, integrou 21 ECA, onde oito estudos incluíam um grupo controlo com placebo e 13 estudos um grupo controlo sem placebo. As análises intention-to-treat (ITT) e per protocol (PP) não mostraram uma diferença estatisti- camente significativa no que concerne à taxa de erradicação do H. pylori entre os doentes com suplementação com probióticos e os doentes do grupo com placebo. Contudo, a taxa de erradicação foi superior no grupo com probióticos comparativamente ao grupo com terapêutica standard isolada $(84,48 \pm 12,61 \%$ e $77,04 \pm 9,4 \%$, respetivamente). Relativamente aos efeitos adversos verificou-se que sintomas como náuseas 


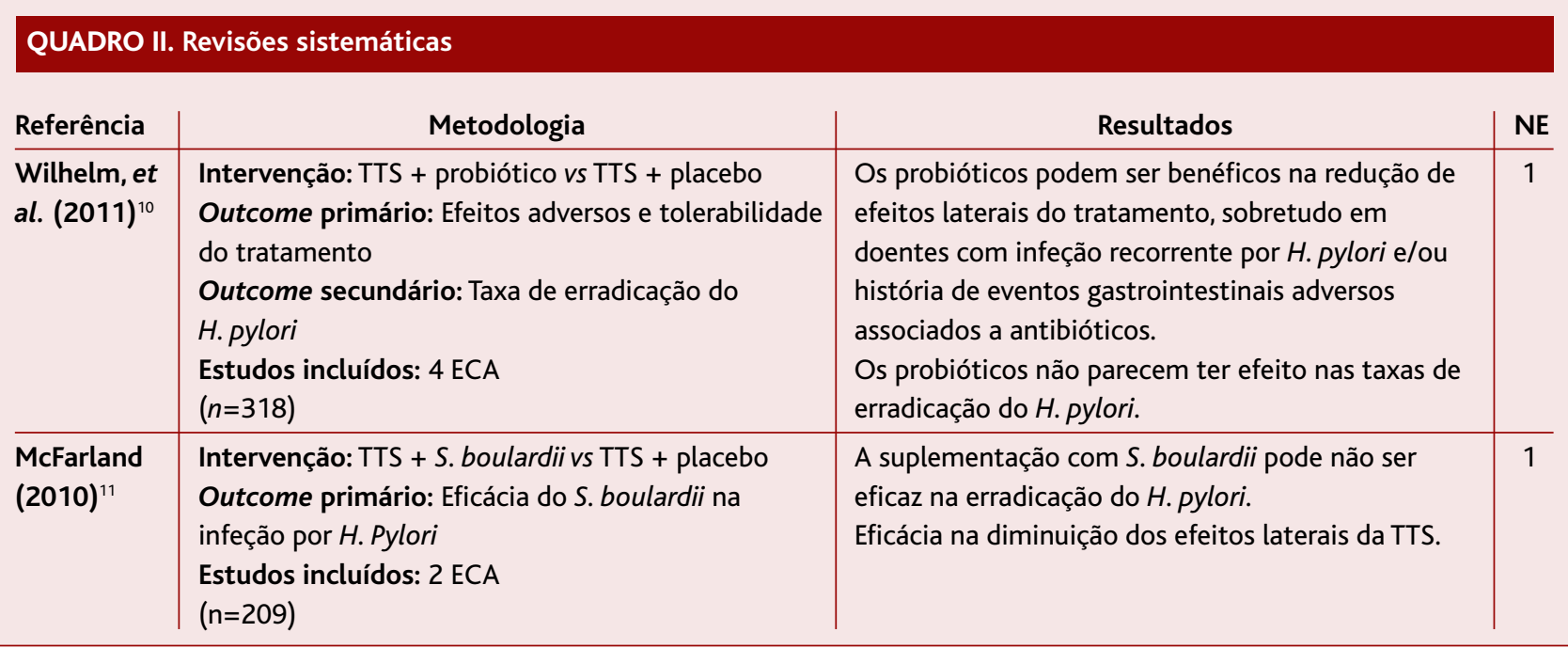

Legenda: $\mathrm{ECA}$ = ensaio clínico aleatorizado e controlado; H. pylori = Helicobacter pylori; NE = nível de evidência; S. boulardii = Saccharomyces boulardii; TTS = terapêutica tripla standard.

(odds-ratio (OR) $=0,43$; intervalo de confiança (IC) 95\%: 0,27-0,7), vómitos ( $\mathrm{OR}=0,3$; IC95\% 0,11-0,86), diarreia $(\mathrm{OR}=0,43$; IC95\% 0,21-0,89) e obstipação (OR=0,28; IC95\% 0,13-0,64) eram melhorados com a suplementação de probióticos comparativamente à terapêutica standard isolada. Quando comparados os grupos com suplementação de probióticos e os grupos com placebo verificou-se que as náuseas (OR=0,36; IC95\% 0,21$-0,62)$, a diarreia $(\mathrm{OR}=0,33$; IC95\% $0,19-0,57)$ e a distensão abdominal (OR=0,5; IC95\% 0,3-0,83) sofriam uma melhoria no grupo com probióticos comparativamente ao grupo com placebo. Os autores concluíram que não existia diferença estatisticamente significativa relativamente entre a taxa de erradicação do $H$. pylori dos grupos suplementados com probióticos e a dos grupos suplementados com placebo, sugerindo que o placebo pode, por si só, influenciar a taxa de erradicação da bactéria. Relativamente aos efeitos adversos associados à terapêutica de erradicação, os probióticos melhoraram claramente as náuseas e a diarreia (NE 2).

Na MA de Zhu e colaboradores, ${ }^{3}$ realizada em 2014, foram incluídos $14 \mathrm{ECA}$, nove com doentes caucasianos e cinco com doentes asiáticos. Nesta MA, a adição de probióticos à terapêutica tripla standard revelou uma taxa de erradicação do H. pylori superior comparativamente à terapêutica tripla standard isolada ou associada a placebo, com o valor de OR na análise ITT e PP de
1,67 (IC95\% 1,38-2,02) e 1,68 (IC95\% 1,35-2,08), respetivamente. De salientar que a sensibilidade dos estudos asiáticos (OR=1,78; IC95\% 1,40-2,26) foi superior à dos estudos caucasianos (OR=1,48; IC95\% 1,06-2,05). Dez dos estudos incluídos na MA forneciam também dados acerca da incidência de efeitos adversos do tratamento. Verificou-se que, no grupo tratado com probióticos, a incidência global de efeitos laterais foi inferior comparativamente ao grupo sem probióticos $(\mathrm{OR}=0,49$; IC95\% 0,26-0,94). Individualmente, a suplementação com probióticos diminuiu de forma significativa a incidência de diarreia (OR=0,21; IC95\% 0,06-0,74). Relativamente a outros efeitos adversos, nomeadamente alterações do paladar, gosto metálico, vómitos, náuseas e dor epigástrica, não se verificou uma diferença significativa entre os dois grupos. Os autores concluíram que a suplementação com probióticos durante o tratamento de erradicação do H. pylori pode ter efeitos benéficos na taxa de erradicação, sobretudo em doentes de etnia asiática, assim como na incidência de efeitos adversos globais, particularmente na diarreia (NE 1).

\section{Revisões sistemáticas}

Apesar de mostrarem um efeito positivo dos probióticos relativamente aos efeitos adversos, as duas RS analisadas não mostraram benefício da sua utilização para a erradicação do H. pylori (Quadro II). 


\begin{tabular}{|c|c|c|c|c|}
\hline Referência & População & Intervenção & Resultados & NE \\
\hline $\begin{array}{l}\text { Tongtawee, } \\
\text { et al. } \\
(2015)^{12}\end{array}$ & $\begin{array}{l}\text { Adultos com } \\
\text { gastrite associada } \\
\text { ao } H \text {. pylori } \\
(n=300)\end{array}$ & $\begin{array}{l}\text { TTS + placebo } 1 \text { sem. vs pré- } \\
\text {-tratamento com probióticos } \\
1 \text { sem. seguida de TTS vs TTS } \\
\text { com } 1 \text { sem. de pré e pós- } \\
\text {-tratamento com probióticos }\end{array}$ & $\begin{array}{l}\text { Taxa de erradicação superior nos grupos com } \\
\text { probióticos. } \\
\text { Incidência dos efeitos laterais sem diferença } \\
\text { significativa entre os grupos com probióticos e o } \\
\text { grupo com placebo. }\end{array}$ & 2 \\
\hline $\begin{array}{l}\text { Tongtawee, } \\
\text { et al. } \\
(2015)^{13}\end{array}$ & $\begin{array}{l}\text { Adultos com } \\
\text { gastrite associada } \\
\text { ao H. pylori } \\
(n=200)\end{array}$ & $\begin{array}{l}\text { Pré-tratamento com placebo } \\
1 \text { sem. seguida de TTS } 1 \text { sem. } \\
\text { vs pré-tratamento com } \\
\text { probióticos } 1 \text { sem. seguida } \\
\text { terapia TTS } 1 \mathrm{sem} .\end{array}$ & $\begin{array}{l}\text { Taxa de erradicação superior no grupo com } \\
\text { pré-tratamento com probióticos em relação ao } \\
\text { grupo com pré-tratamento com placebo. } \\
\text { Incidência dos efeitos laterais sem diferença } \\
\text { significativa entre os grupos. }\end{array}$ & 2 \\
\hline $\begin{array}{l}\text { Ma, et al. } \\
(2015)^{14}\end{array}$ & $\begin{array}{l}\text { Adultos com } \\
\text { úlcera péptica e } \\
\text { infeção por } \\
\text { H. pylori } \\
(n=132)\end{array}$ & $\begin{array}{l}\text { TTS + probióticos vs TTS } \\
+ \text { placebo }\end{array}$ & $\begin{array}{l}\text { Taxa de erradicação superior no grupo com } \\
\text { probióticos em relação ao grupo com placebo. } \\
\text { Diminuição dos efeitos laterais no grupo com } \\
\text { probióticos. }\end{array}$ & 2 \\
\hline $\begin{array}{l}\text { Hauser, et } \\
\text { al. }(2015)^{15}\end{array}$ & $\begin{array}{l}\text { Adultos com } \\
\text { infeção por } \\
\text { H. pylori } \\
(n=650)\end{array}$ & $\begin{array}{l}\text { TTS + probióticos vs TTS } \\
+ \text { placebo }\end{array}$ & $\begin{array}{l}\text { Taxa de erradicação superior no grupo com } \\
\text { probióticos em relação ao grupo com placebo. } \\
\text { Diminuição dos efeitos laterais no grupo com } \\
\text { probióticos. }\end{array}$ & 1 \\
\hline $\begin{array}{l}\text { Francavilla, } \\
\text { et al. } \\
(2014)^{16}\end{array}$ & $\begin{array}{l}\text { Adultos com } \\
\text { infeção por } \\
\text { H. pylori } \\
(n=100)\end{array}$ & $\begin{array}{l}\text { TTS + probióticos com período } \\
\text { pré e pós-tratamento com } \\
\text { probióticos vs TTS + placebo } \\
\text { com período pré e pós- } \\
\text {-tratamento com placebo }\end{array}$ & $\begin{array}{l}\text { Taxa de erradicação superior no grupo com } \\
\text { probióticos em relação ao grupo placebo (diferença } \\
\text { estatisticamente não significativa). } \\
\text { Redução significativa dos efeitos laterais no grupo } \\
\text { com probióticos. }\end{array}$ & 2 \\
\hline
\end{tabular}

Legenda: $H$. pylori = Helicobacter pylori; NE = nível de evidência; sem. = semana; TTS = terapêutica tripla standard .

A RS de Wilhelm e colaboradores, ${ }^{10}$ realizada em 2011, englobou quatro ECA. Em três dos estudos incluídos verificou-se que os probióticos aumentavam a tolerabilidade e diminuíam os efeitos adversos do tratamento de erradicação do H. pylori, particularmente as náuseas, alterações do paladar, diarreia e dor epigástrica. Apenas um estudo não apresentou os mesmos resultados; contudo, era o estudo que reunia uma amostra mais pequena e utilizava um suplemento com probióticos à base de leite. Em todos os estudos, os probióticos não mostraram efeitos significativos na taxa de erradicação do H. pylori (NE 1). De salientar que todos os estudos incluídos nesta RS utilizaram diferentes regimes de erradicação e diferentes probióticos. Os autores alertam ainda para o facto de o esquema terapêutico ter tido uma duração de apenas sete dias, o que pode ter influenciado os resultados, principalmente no que concerne à taxa de erradicação da bactéria.

A outra revisão, de McFarland, ${ }^{11}$ realizada em 2010, teve como objetivo rever a evidência acerca da eficácia e segurança do $S$. boulardii para diversas patologias. Relativamente à infeção por $H$. pylori foram incluídos dois ECA. Em ambos os estudos não se verificou uma diferença significativa no que concerne à taxa de erradicação do H. pylori entre o grupo suplementado com probióticos e o grupo placebo. Contudo, o grupo suplementado com probiótico teve uma menor incidência de efeitos laterais, nomeadamente diarreia, dor epigástrica e dispepsia (NE 1).

\section{Ensaios clínicos aleatorizados}

Relativamente aos ensaios clínicos, todos os ECA in- 
QUADRO IV. Normas de orientação clínica

\begin{tabular}{|c|c|c|}
\hline Referência & Recomendações & NE/FR \\
\hline $\begin{array}{l}\text { University of Texas School } \\
\text { of Nursing Family Nurse } \\
\text { Practitioner Program (2013) }{ }^{17}\end{array}$ & $\begin{array}{l}\text { A suplementação com probióticos (S. boulardii) pode ser considerada, uma vez que } \\
\text { parece aumentar as taxas de erradicação do } H \text {. pylori e diminuir os efeitos } \\
\text { adversos do tratamento (diarreia). }\end{array}$ & $\mathrm{FR} \mathrm{A}^{*}$ \\
\hline Hungin, et al. (2013) ${ }^{18}$ & $\begin{array}{l}\text { Determinados probióticos são úteis como terapêutica adjuvante na prevenção } \\
\text { ou redução da duração/intensidade da diarreia associada ao tratamento. }\end{array}$ & NE Alto ${ }^{\dagger}$ \\
\hline $\begin{array}{l}\text { The Maastricht IV/Florence } \\
\text { Consensus Report (2012) })^{19}\end{array}$ & $\begin{array}{l}\text { Alguns probióticos apresentam resultados promissores na redução de efeitos } \\
\text { laterais como adjuvantes no tratamento de erradicação do } H \text {. pylori. }\end{array}$ & $\mathrm{FR} \mathrm{D}^{\ddagger}$ \\
\hline
\end{tabular}

Legenda: FR = Força de recomendação; $H$. pylori = Helicobacter pylori; NE = Nível de evidência; S. boulardii = Saccharomyces boulardii.

* FR atribuída pela NOC com base no U. S. Preventive Services Task Force (USPSTF) Ratings. ${ }^{17}$

† NE atribuído pela NOC com base no Grades of Recommendation Assessment, Development and Evaluation (GRADE) System. ${ }^{18}$

₹ FR atribuída pela NOC com base no sistema elaborado para o Maastricht III Consensus Report. ${ }^{19}$

cluídos nesta revisão tinham como outcomes a taxa de erradicação do $H$. pylori e a presença de efeitos laterais do tratamento. De uma forma global, os cinco ECA evidenciaram taxas de erradicação da bactéria superiores no grupo medicado com probióticos (Quadro III).

O ECA de Tongtawee e colaboradores, ${ }^{12}$ de 2015, englobou 300 doentes com diagnóstico de gastrite associada ao H. pylori que foram randomizados em três grupos. As opções terapêuticas testadas consistiram em: (1) terapêutica tripla (esomeprazol 20mg bid + claritromicina $500 \mathrm{mg} /$ metronidazol $400 \mathrm{mg}$ bid + amoxicilina $1000 \mathrm{mg}$ bid) associada a placebo, durante uma semana; (2) pré-tratamento durante uma semana com probióticos, contendo Lactobacillus delbrueckii subsp. bulgaricus e Streptococcus thermophilus, seguido de terapêutica tripla; e (3) terapêutica tripla com uma semana de pré e pós-tratamento com probióticos. A taxa de erradicação do H. pylori foi superior nos grupos que incluíram probióticos no esquema terapêutico comparativamente ao grupo tratado com placebo, tanto pela análise ITT $(p<0,01$; IC95\% 0,72-0,87) como PP ( $p<0,01$; IC95\% 0,71$-0,97)$. Relativamente aos efeitos laterais, o grupo tratado com placebo apresentou uma maior incidência dos mesmos; contudo, a diferença entre os três grupos não se revelou estatisticamente significativa $(p=0,32)$. De acordo com os critérios da escala SORT trata-se de um ECA de qualidade limitada, pelo que se atribui um NE 2.

Tongtawee e colaboradores ${ }^{13}$ realizaram outro ECA, em 2015, muito semelhante ao anterior. Este estudo englobou 200 doentes com diagnóstico de gastrite as- sociada ao H. pylori. Estes foram randomizados em dois grupos. Um dos grupos foi tratado com uma semana de placebo, seguida de uma semana de tratamento com terapêutica tripla (esomeprazol $20 \mathrm{mg}$ + claritromicina $500 \mathrm{mg} /$ metronidazol 400mg + amoxicilina $1000 \mathrm{mg}$, todos duas vezes por dia); o outro grupo fez o mesmo esquema de terapêutica tripla, com pré-tratamento de uma semana com probióticos, contendo Lactobacillus delbrueckii subsp. bulgaricus e Streptococcus thermophilus. De acordo com as análises ITT e PP, a taxa de erradicação do $H$. pylori foi superior no grupo tratado com probióticos comparativamente ao grupo placebo ( $p=0,01$; IC95\% 0,04-0,15 e $p=0,04$; IC95\% 0,02-0,13, respetivamente). No que diz respeito aos efeitos adversos do tratamento não se observou uma diferença estatisticamente significativa entre os dois grupos. De acordo com os critérios da escala SORT trata-se de um ECA de qualidade limitada, pelo que se atribui um NE 2.

O ECA de MA e colaboradores,$^{14}$ levado a cabo em 2015, envolveu 132 doentes com diagnóstico de úlcera péptica e infeção por $H$. pylori. Os pacientes foram randomizados para receber terapêutica tripla (omeprazol $20 \mathrm{mg}$ uma vez por dia + claritromicina 500mg duas vezes por dia $+400 \mathrm{mg}$ de metronidazole duas vezes por dia) associada a probióticos ou a placebo. Verificou-se que a taxa de erradicação do $H$. pylori foi superior no grupo com esquema terapêutico que incluía probióticos comparativamente ao grupo tratado apenas com terapêutica tripla e placebo $(p<0,05)$. No que diz respeito aos efeitos adversos do tratamento verificou-se que 
a incidência de efeitos adversos foi significativamente menor no grupo suplementado com probióticos em relação ao grupo com placebo $(p<0,05)$. De acordo com os critérios da escala SORT trata-se de um ECA de qualidade limitada, pelo que se atribui um NE 2.

O estudo de Hauser e colaboradores, ${ }^{15}$ elaborado em 2015, incluiu uma amostra de 804 doentes provenientes de diferentes centros médicos que foram randomizados em dois grupos. Ambos os grupos receberam a terapêutica tripla standard de erradicação do H. pylori, suplementada com probióticos (contendo Lactobacillus rhamnosus, L. rhamnosus e Bifidobacterium) ou com placebo. O esquema terapêutico teve uma duração de 14 dias. Os autores alertam para o facto de a terapêutica tripla não ter sido a mesma para todos os doentes, uma vez que a escolha do esquema de erradicação foi da responsabilidade de cada centro. A análise final englobou 650 doentes. Verificou-se uma taxa de erradicação superior no grupo de doentes suplementado com probióticos (87,38\%; IC95\% 84,33-90,21\%) relativamente ao grupo com placebo (72,55\%; IC95\% 69,65-75,81\%) (OR=2,62; IC95\% 1,71-4,02).Verificou-se ainda uma melhoria global dos sintomas no grupo com probióticos, principalmente em relação à dor epigástrica $(P=0,039)$, distensão abdominal $(P=0,022)$, flatulência $(P<0,001)$, alterações do paladar $(P=0,002)$, náuseas $(P<0,001)$, azia $(P<0,001)$ e diarreia $(P<0,001)$. No que diz respeito aos efeitos laterais do tratamento, a intensidade média de sintomas adversos potencialmente associados à terapêutica antibiótica foi significativamente maior no grupo com placebo $(0,76 v s 0,55 ; p<0,001)$. De acordo com os critérios da escala SORT trata-se de um ECA de boa qualidade, pelo que se atribui um NE 1.

Francavilla e colaboradores ${ }^{16}$ realizaram, em 2014, um ECA cujo objetivo era testar todos os possíveis efeitos de uma preparação com duas estirpes de Lactobacillus reuteri (L. reuteri) nos indivíduos com infeção por H. pylori. A amostra era constituída por 100 doentes que foram randomizados em dois grupos: um grupo com terapêutica de erradicação, constituída por inibidor da bomba de protões, claritromicina e amoxicilina, durante sete dias, suplementado com preparação de probiótico e o outro grupo com terapêutica de erradicação e placebo. Tanto o probiótico como o placebo foram administrados durante as três fases do estudo: pré-erradicação (dia 0-28); erradicação (dia 29-35); e fol- low-up (dia 36-96). De acordo com as análises ITT e PP, no final do período de follow-up a taxa de erradicação foi superior no grupo com probiótico comparativamente ao grupo placebo (OR=1,5; IC95\% 0,6-0,39 e OR=1,6; IC95\% 0,6-0,41, respetivamente); contudo, esta diferença não foi estatisticamente significativa. No que concerne aos efeitos adversos da terapêutica durante a fase de erradicação verificou-se uma redução da incidência dos mesmos no grupo suplementado com a combinação de L. reuteri, nomeadamente da dor e distensão abdominal $(p<0,04)$ e diarreia $(p<0,05)$. De acordo com os critérios da escala SORT trata-se de um ECA de qualidade limitada, pelo que se atribui um NE 2.

\section{Normas de orientação clínica}

De uma forma geral, todas as NOC analisadas consideram que os probióticos podem ter um papel no tratamento de erradicação do H. pylori, nomeadamente no que diz respeito à redução dos efeitos adversos a ele associados (Quadro IV). ${ }^{17-19}$

A NOC da University of Texas School of Nursing Family Nurse Practitioner Program ${ }^{17}$ (de 2013) estabelece que a suplementação da terapêutica tripla standard com probióticos, como S. boulardii, pode ser considerada uma vez que parece aumentar as taxas de erradicação do $H$. pylori e diminuir os efeitos adversos do tratamento, nomeadamente da diarreia. Esta norma atribui a esta recomendação uma FR A, indicando existir uma grande certeza de que os benefícios são substanciais e de que esta terapêutica adjuvante deve ser oferecida. ${ }^{17}$

Por outro lado, de acordo com as recomendações de Hungin e colaboradores ${ }^{18}$ (de 2013), em doentes sob terapêutica de erradicação do $H$. pylori determinados probióticos, como o S. boulardii, o L. rhamnosus GG e outras combinações de probióticos comercializadas, são úteis como terapêutica adjuvante na prevenção ou redução da duração e/ ou intensidade da diarreia associada ao tratamento. A esta recomendação, realizada com base em quatro ensaios aleatorizados, controlados e duplamente cegos, com um total de 382 pacientes, foi atribuído um NE alto, considerando-se pouco provável que investigação futura altere a evidência encontrada. ${ }^{18}$

O Maastricht IV/Florence Consensus Report ${ }^{19}$ (de 2012) considera que determinados prebióticos e probióticos mostram resultados promissores como terapêutica adjuvante no que concerne à diminuição dos 
efeitos laterais do tratamento de erradicação do $H$. $p y$ lori (FR D). Esta recomendação traduz essencialmente a opinião dos especialistas envolvidos no grupo de trabalho deste Consenso, uma vez que este considerou que a má qualidade de muitos dos ensaios clínicos analisados e a grande heterogeneidade entre eles limitavam a atribuição de uma recomendação mais forte. ${ }^{19}$

\section{CONCLUSÕES}

De acordo com a evidência atual disponível pode concluir-se que a adição de probióticos ao esquema de tratamento utilizado para a erradicação do H. pylori pode ser benéfica, uma vez que parece associar-se a taxas de erradicação superiores (FR B) com menores efeitos adversos associados (FR A).

A maioria dos estudos incluídos nesta revisão demonstrou uma taxa de erradicação do H. pylori superior nos doentes em que os probióticos estavam incluídos no esquema terapêutico, comparativamente com o grupo controlo. A única NOC incluída que fazia referência à taxa de erradicação, a do University of Texas School of Nursing Family Nurse Practitioner Program, ${ }^{17}$ recomenda o seu uso com uma FR A. Uma MA, com NE 2, não encontrou evidência suficiente que suporte a recomendação do uso de probióticos com o objetivo de aumentar a taxa de erradicação do H. pylori, assim como duas RS com NE 1. É apenas de salientar que numa das RS a duração do esquema terapêutico foi de sete dias, o que pode ter influenciado os resultados.

No que concerne à redução dos efeitos laterais do esquema de erradicação do H. pylori, a maioria dos estudos é unânime acerca do benefício da adição dos probióticos.

Não é claro se os probióticos têm um papel direto no aumento da taxa de erradicação do H. pyloriou se esse resultado se deve à diminuição dos efeitos adversos do tratamento, que proporciona uma maior adesão ao mesmo.

Apesar da globalidade dos estudos constituírem estudos bem desenhados, estes possuem limitações. Em primeiro lugar, verificou-se a existência de uma grande heterogeneidade das espécies de probióticos incluídas nos diferentes estudos, não permitindo uma comparação direta e uma recomendação formal acerca da estirpe mais adequada a ser utilizada como adjuvante no esquema de erradicação. Os próprios esquemas de erradicação utilizados foram também diferentes, assim como a duração da terapêutica, o que pode ter tido influência na taxa de erradicação e tolerabilidade do tratamento. Por outro lado, a análise dos efeitos adversos foi, em muitos estudos, avaliada por questionário, o que pode ter enviesado os resultados dada a natureza subjetiva inerente a este método de colheita e a inevitável variabilidade da resposta dos doentes aos efeitos adversos. Salienta-se ainda que os estudos incluídos na revisão não foram realizados para a população portuguesa, apesar de a maioria utilizar os antimicrobianos que são recomendados na Norma de Orientação Clínica da Direção-Geral da Saúde para a erradicação do H. pylori. ${ }^{20}$

Assim sendo, apesar do papel dos probióticos como adjuvantes à terapêutica standard no que concerne à taxa de erradicação do H. pylori necessitar ainda de validação através de estudos com metodologia mais homogénea, o seu benefício na diminuição dos efeitos laterais do tratamento é inequívoco. Desta forma, considera-se benéfica a sua adição ao esquema de erradicação do $H$. pylori.

\section{REFERÊNCIAS BIBLIOGRÁFICAS}

1. Pounder RE, Ng D. The prevalence of Helicobacter pylori infection in different countries. Aliment Pharmacol Ther. 1995;9 Suppl 2:33-9.

2. IARC Working Group on the Evaluation of Carcinogenic Risk to Humans. Schistosomes, liver flukes and Helicobacter pylori [Internet]. Lyon: International Agency for Research on Cancer; 1994. Available from: https://www. ncbi.nlm.nih.gov/books/NBK487782/

3. Zhu R, Chen K, Zheng YY, Zhang HW, Wang JS, Xia YJ, et al. Meta-analysis of the efficacy of probiotics in Helicobacter pylori eradication therapy. World J Gastroenterol. 2014;20(47):18013-21.

4. Vakil N. Helicobacter pylori: factors affecting eradication and recurrence. Am J Gastroenterol. 2005;100(11):2393-4.

5. Laheij RJ, Rossum LG, Jansen JB, Straatman H, VerbeekAL. Evaluation of treatment regimens to cure Helicobacter pylori infection: a meta-analysis. Aliment Pharmacol Ther. 1999;13(7):857-64.

6. Dang Y, Reinhardt JD, Zhou X, Zhang G. The effect of probiotics supplementation on Helicobacter pylori eradication rates and side effects during eradication therapy: a meta-analysis. PLoS One. 2014;9(11):e111030.

7. Food and Agriculture Organization of the United Nations, World Health Organization. Probiotics in food: health and nutritional properties and guidelines for evaluation [Internet]. Rome: FAO/WHO; 2006. Available from: http://www. fao.org/tempref/docrep/fao/009/a0512e/a0512e00.pdf

8. Ebell MH, Siwek J, Weiss BD, Woolf SH, Susman J, Ewigman B, et al. Strength of recommendation taxonomy (SORT): a patient-centered approach to grading evidence in the medical literature. Am Fam Physician. 2004;69(3):54856.

9. Lu C, Sang J, He H, Wan X, Lin Y, Li L, et al. Probiotic supplementation does not improve eradication rate of Helicobacter pylori infection compared to placebo based on standard therapy: a meta-analysis. Sci Rep. 2016;6:23522.

10. Wilhelm SM, Johnson JL, Kale-Pradhan PB. Treating bugs with bugs: the role of probiotics as adjunctive therapy for Helicobacter pylori. Ann Pharmacother. 2011;45(7-8):960-6.

11. McFarland LV. Systematic review and meta-analysis of Saccharomyces bou- 
lardii in adult patients. World J Gastroenterol. 2010;16(18):2202-22.

12. Tongtawee T, Dechsukhum C, LeeanansaksiriW, Kaewpitoon S, Kaewpitoon $\mathrm{N}$, Loyd RA, et al. Improved Helicobacter pylori eradication rate of tailored triple therapy by adding Lactobacillus delbrueckii and Streptococcus thermophilus in Northeast Region of Thailand: a prospective randomized controlled clinical trial. Gastroenterol Res Pract. 2015;2015:518018.

13. Tongtawee T, Dechsukhum C, Leeanansaksiri W, Kaewpitoon S, Kaewpitoon $\mathrm{N}$, Loyd RA, et al. Effect of pretreatment with Lactobacillus delbrueckii and Streptococcus thermophillus on tailored triple therapy for Helicobacter pylori eradication: a prospective randomized controlled clinical trial. Asian Pac J Cancer Prev. 2015;16(12):4885-90.

14. Ma F, Zhou C, Wang J, Liu T, Liu J. Probiotics in the treatment of peptic ulcer infected by helicobacter pylory and its safety. Pak J Pharm Sci. 2015;28(3 Suppl):1087-90.

15. Hauser G, Salkic N, Vukelic K, Jajacknez A, Stimac D. Probiotics for standard triple Helicobacter pylori eradication: a randomized, double-blind, placebocontrolled trial. Medicine (Baltimore) 2015;94(17):e685.

16. Francavilla R, Polimeno L, Demichina A, Maurogiovanni G, Principi B, Scaccianoce $G$, et al. Lactobacillus reuteri strain combination in Helicobacter pylori infection: a randomized, double-blind, placebo-controlled study. J Clin Gastroenterol. 2014;48(5):407-13.

17. University of Texas at Austin, School of Nursing Family Nurse Practitioner Program. Recommendations in primary care for the most efficacious and cost effective pharmacologic treatment for Helicobacter pylori in non-pregnant adults [Internet]. National Guideline Clearinghouse; 2013 May [updated 2016 May 18]. Available from: https://www.guideline.gov/summa- ries/summary/46427

18. Hungin AP, Mulligan C, Pot B, Whorwell P, Agréus L, Fracasso P, et al. Systematic review: probiotics in the management of lower gastrointestinal symptoms in clinical practice - an evidence-based international guide. Aliment Pharmacol Ther. 2013;38(8):864-86.

19. Malfertheiner P, Megraud F, O'Morain CA, Atherton J, Axon AT, Bazzoli F, et al. Management of Helicobacter pylori infection: the Maastricht IV/ Florence Consensus Report. Gut. 2012;61(5):646-64.

20. Direção-Geral da Saúde. Supressão ácida: utilização dos inibidores da bomba de protões e das suas alternativas terapêuticas - norma nº 036/2011, de 30/09/2011. Lisboa: DGS; 2011.

\section{CONFLITO DE INTERESSES}

As autoras declaram não ter quaisquer conflitos de interesse.

\section{FINANCIAMENTO DO ESTUDO}

As autoras declaram que o trabalho relatado neste manuscrito não foi objeto de qualquer tipo de financiamento externo.

\section{ENDEREÇO PARA CORRESPONDÊNCIA \\ Cristiana Sousa Pinto \\ E-mail: cristianapinto@hotmail.com \\ https://orcid.org/0000-0002-0897-3750}

Recebido em 22-12-2017

Aceite para publicação em 14-09-2018

\section{ABSTRACT}

\section{THE EFFECT OF PROBIOTICS ON HELICOBACTER PYLORI ERADICATION: AN EVIDENCE-BASED REVIEW}

Introduction: Helicobacter pylori (H. pylori) infection is an important risk factor for several gastric diseases, including peptic ulcer, chronic atrophic gastritis and gastric cancer, and so its eradication is the main therapeutic goal. Probiotics can help to achieve this goal, and equally play a positive role in reducing the side effects of the treatment.

Objetive: To determine the effect of probiotics as an adjuvant therapy for $\mathrm{H}$. pylori eradication in adults.

Data sources: MEDLINE and evidence-based medicine sites.

Revision methods: A research of meta-analyses (MA), systematic reviews (SR), randomised controlled trials (RCTs) and clinical practice guidelines (CPG) was performed using the MeSH terms 'Helicobacter pylori' and 'Probiotics'. The research was limited to articles published between January 2006 and July 2016, in English and Portuguese. The Strength of Recommendation Taxonomy was used to assign the level of evidence and the strength of recommendation.

Results: A total of 61 articles was found; amongst these, two MAs, two RSs, five RCTs and three CPGs met the inclusion criteria and were therefore selected. MAs present mixed results: one showed higherr eradication rates with probiotic treatment; the other did not demonstrate superiority of probiotics when compared with placebo. Both showed its beneficial effect in reducing side effects. In spite of showing a positive effect of probiotics on adverse effects, the two SRs did not show benefit of their use for the eradication of $\mathrm{H}$. pylori. All RCTs showed higher bacterial eradication rates in the group treated with probiotics. CPGs suggest the use of probiotics in the treatment of $\mathrm{H}$. pylori eradication.

Conclusions: Adding probiotics to the treatment regimen used for $\mathrm{H}$. pylori eradication may be beneficial since it seems to be associated with higher eradication rates (strength of recommendation B) and less adverse effects (strength of recommendation A).

Keywords: Helicobacter pylori; Probiotics. 